\title{
Subject Review: Machine Learning and Deep Learning based Arabic Handwriting Recognition
}

\author{
AHMED KAWTHER HUSSEIN \\ Department of Computer Science, College of Education, MustansiriyahUniversity \\ Baghdad-Iraq
}

\begin{abstract}
In recent times, with the progress of artificial intelligence and machine learning, handwriting recognition becomes an active research area. Furthermore, many products of applications have provided handwriting recognition services in various languages with a low margin of errors. The Arabic language has its challenges which make it not easy comparing with other languages to develop error-free and practical Arabic handwriting recognition. Deep learning has promising results in this field. This paper surveys existing literature work for Arabic handwriting recognition in general and using deep learning in particular.
\end{abstract}

Key Words: Handwriting recognition, Arabic handwriting recognition, Machine learning, Deep learning, Natural language processing, Neural network.

\section{INTRODUCTION}

Handwriting recognition is considered one of the top research topics in the field of machine learning. Its goal is to enable smart systems to read human writing and recognition it accurately similar to human beings. Typically, texts are considered to be handwritten, then converted to electronic documents using scanners, cameras, or video recorders. recordings [1,2]. One of the most essential applications of handwriting recognition is enabling a comfortable way for human smart machine interaction, and human-human interaction using smart machines such as smartphones, tablets, or pocket PC. Such devices represent the new trend of communication as well as the portable personal PC of everyone. However, their small size prevents a comfortable and easy way of writing text in it. Using the digital pen became more practical and preferable to people. The only hurdle in this is enabling the device to convert handwritten text to typed text to make it interpreted. The efforts of enabling the smart device to read a handwritten text and convert it to a typical typed text have existed for more than 30 years, however, it became the recent years more feasible and its providing more promising results. Shortly, digital pens will become a more practical and ergonomic humanmachine interface than the keyboard for any language including the most difficult interpretable language scripts [3]. Another important application of handwriting recognition is the conduction of manuscripts editing which has some challenges related to image processing, image quality degradation; however, it is arguably simpler due to its higher organization of writing than freestyle of writing.

The Arabic language is the communication language among 223 million and an essential cultural language by many more. The Arabic language has numerous dialects according to the region, however, the standard version of Arabic or what is named as modern standard Arabic is used for formal communication for all Arabic speakers. Furthermore, the Arabic content existence and the Arabic contribution to the internet is widely recognizable. These facts make the topic of Arabic handwriting recognition as an essential topic. Talking about Arabic handwriting recognition leads to identifying various challenges: Arabic script is curved for many letters and has a continuity nature. Also, many Arabic letters are similar in writing with small differences. However, the characteristics of Arabic letters are similar to a set of other languages like Farsi, Urdu, and others which makes the process of Arabic handwriting recognition has widespread benefits as well as can take help from other languages' efforts of handwriting recognition $[4,3]$.

In recent times machine learning models have witnessed an emerging and it has attracted researchers due to its extraordinary and promising results [5]. Deep learning is a sub-field of machine learning that has attracted the special attention of researchers [6]. One particular type of machine learning models, namely, deep learning, has gained an increasing interest in the recent time due to the publication of highly influential articles such as $[7,8,9,10]$. From that time, deep learning became the front page in 
New York times and the research community accepted the idea of training neural networks with multiple layers for generating effective performance. Furthermore, the emerging in computer hardware and data makes it more feasible than any time before.

The literature contains many surveys focusing on Arabic handwriting recognition, however, non-of them has paid attention to deep learning-based models for Arabic handwriting recognition which is the goal of this article. The remaining of the article is organized as follows. In section-2-, we present the general taxonomy of handwriting recognition. Next, in section -3- we provide a summary of previous reviews for Arabic handwriting recognition. Section -4- for handwriting recognition, gives an overview of general models. Sections -5- introduce the review of the Arabic handwriting recognition from different perspectives, with more focus on deep learning-based models. Section -6- presents the summary and open challenges.

\section{HANDWRITIMG RECOGNITION - TAXONOMY}

Handwriting recognition can be categorized from different angles of view [11]. The nature of the application: online or off-line, the source of data: natural seen, scanned document, or manuscript, the content of data: digits, words, or text.

- Offline Recognition: the writing is performed and then provided to the computer system for recognition.

- Online Recognition: the writing is provided for recognition while doing the writing, this means the time series of every generation of the pixel in the writing is provided.

- natural seen: the writing is part of an image that is taken from natural seen such as signboard or advertisement

- $\quad$ scanned document: the writing is from a scanned document that contains text written by today's tools

- manuscript: the writing is from a scanned old historical document, a degradation of the image quality and differences in today 's way might occur

- Digit writing: the data represent a digit which makes it limited in the classes of prediction or recognition

- Words writing: the data represent words, which is a combination of different characters, however, each word is a separate class.

- Text writing: the data is a combination of words and characters.

\section{SURVEYS OF ARABIC HANDWRITIMG RECOGNITION}

The literature contains few proper surveys of Arabic handwriting recognition. We mention here the work of [12] that provides a survey on Arabic online handwriting recognition for the past few years. The survey was focused on online approaches without much analysis of them and without presenting the contribution of deep learning in elevating the technology of Arabic handwriting. Another interesting survey is the work of [13] that is focused on off-line handwritten Arabic character recognition. This survey has addressed the challenges of Arabic handwriting systems and provided a review of various approaches carried out for this target.

For the best of our knowledge, this survey is the first in presenting Arabic handwriting recognition from the perspective of machine learning in general and deep learning in particular without limiting the scope to either online or offline models.

\section{MODS FOR HANDWRITIMG RECOGNITION}

The literature of handwriting recognition is huge and it contains a wide range of approaches. In this section, we present some of them. Some researchers have used an integration between deep neural networks and hidden Markov models or model (DNN-HMM) that was applied for handwritten Chinese text recognition. Another work that was based on Hidden Markov models is the work of [14] which aimed at Online Farsi Handwritten Character Recognition. Motivated by the great success of convolutional neural networks CNNs on image net [15] have employed it to calculate its n-gram frequency profile, which is the of n-grams contained set in the word. They calculated Frequencies forbigrams and trigrams for the entire world and its parts.For matching dictionary of whole words used to estimate profile to the true profiles, Correlation Analysis was used. In another work, the Gaussian Mixture Model and SVM classification framework were employed together by [16]. In their work, the Gaussian Mixture Model (GMM) was used to derive a set of features based on character class-conditional probabilities (posterior features). The role is to serve in the discrimination of various classes. [17] a sequential segmentation recognition of Arabic handwriting matching was introduced by starting with a testing set of segmentation widths to get the best segmentation of Arabic letters from the word. An integration between wavelet energy feature (WEF) and extreme learning machine (ELM) for the recognition of handwritten Malayalam character was proposed by [18]. (WEF) was used for feature extraction while ELM for classification. 
International Journal of Engineering Research And Advanced Technology, Vol.5, Issue 10, October-2019

\section{ARABIC HANDWRITIMG RECOGNITION}

Arabic language is spoken by more than 300 million as their native language and is a second language for a wide range of people in the Islamic world as the language of the Quran, the holy book of Muslims. The Arabic language is similar in letters to Farsi and ), Pashto, Jawi, and Kurdish.

The Arabic language is composed of 28 letters. Letters in the Arabic language consist of strokes and dots and they differ in writing according to their positions in the word. We provide the Arabic letters in table-.

Table1.1. Arabic letters and their changes according to the position in the word

\begin{tabular}{|c|c|c|c|}
\hline Initial & Medial & Final & isolated \\
\hline 1 & 1 & 1 & 1 \\
\hline ب & بـ & ب & ب \\
\hline 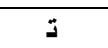 & ت & ت & ت \\
\hline$\dot{\$}$ & $\dot{3}$ & $\dot{H}$ & $\dot{H}$ \\
\hline ج & ج & ج & ج \\
\hline$\Delta$ & $\Delta$ & ج & $\tau$ \\
\hline$\dot{\mathbf{\Delta}}$ & $\dot{\mathbf{\Delta}}$ & خ & $\dot{\tau}$ \\
\hline د & د & د & د \\
\hline$\dot{j}$ & $\dot{j}$ & $\dot{j}$ & $\dot{j}$ \\
\hline J & J & J & J \\
\hline j & $j$ & j & j \\
\hline سد & سد & س & س س \\
\hline ش ش & ش ش & ش ش & ش ش \\
\hline صد & صد & ص & ص \\
\hline ضد & ضد & ض & ض \\
\hline$b$ & $b$ & $b$ & $b$ \\
\hline ظ & ظ & ظ & ظ \\
\hline$s$ & 2 & $\varepsilon$ & $\varepsilon$ \\
\hline$\dot{~}$ & $\dot{\dot{z}}$ & $\dot{\varepsilon}$ & $\dot{\varepsilon}$ \\
\hline$\dot{9}$ & $\dot{\mathbf{g}}$ & ف & ف \\
\hline ق & ق & ق & ق \\
\hline$s$ & $s$ & ك5 & ك5 \\
\hline J & J & J & J \\
\hline مد & هـ & p & 5 \\
\hline$\dot{j}$ & $\dot{j}$ & $\dot{ن}$ & $\dot{ن}$ \\
\hline
\end{tabular}




\begin{tabular}{|c|c|c|c|}
\hline$\Delta$ & $f$ & $d, \ddot{d}$ & ة \\
\hline و & و & و & و \\
\hline 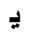 & $\Rightarrow$ & ي & ي \\
\hline
\end{tabular}

\section{DATASETS}

The IFN/ENIT-database: In the work of [19], this dataset was composed of 411 writers. It contains 26400 names and 210000 characters. The ground truth information is embedded in the data. this dataset used for The Arabic handwriting recognition competition held at International Conference on Document Analysis and Recognition (ICDAR) [20].

KHATT database [21] In 2012, KFUPM Handwritten Arabic TexT (KHATT) was presented in the conference of ICFHR. 1000 writers have participated in the writing. The writers are from 18 countries and the data was gathered by 46 sources. It is composed of 2000 paragraphs. This dataset is considered the most comprehensive dataset that can be used for many applications in addition to handwriting recognition such as writer identification.

\section{MACHINE LEARNING MODELS FOR ARABIC HANDWRITIMG RECOGNITION}

In the study of [22], an approach for providing the shape of Arabic handwritten characters. The work has combined both statistical and structural approaches. Modified direction features and support vector machine was used to recognize the exact shape of a character, Dot-descriptors are used.

The work of [23] integrates deep neural networks with hidden Markov model DNN-HMM hybrid systems. Three types were compared: Bi-Directional Long Short-term Memory, (TDNN) is Time Delay Neural Network and LSTM. The second stage is based on HMM and integrates a context-dependent character modeling. KHATT database was used for evaluation.

In the work of [21], Arabic handwriting recognition was implemented using (LSTM) is Long Short-Term Memory networks. The final layer has used Connectionist Temporal Classification (CTC) to enable alignment between the predicted labels and the most suitable path. The application of Multi-Dimensional Long Short-Term Memory networks scans text-lines in all directions to cover fine inflammation in the horizontal and vertical direction.

The authors of [24] propose a new system based on deep recurrent neural networks on which the dropout technique was applied. due to their recurrent connections, Their approach was practical in sequence modeling.furthermore,because of many non-linear hidden layers, it can learn the intricate relationship between input and output layers. In addition to these contributions, due to the powerful performance of dropout, the system is protected against overfitting. This system was tested with a large dataset ADAB to display its performance against difficult conditions like the variety of the large vocabulary and diversity of style, writers.

Some researchers such as [1] have used multi-stage HMM-based handwriting recognition. core shapes were separated by the diacritics and then represented by smaller units called as sub-core shapes. This provided reductions in the number of models that need to be trained. Contextual HMM modeling utilizing. It leads to the significantly compact recognizer. Moreover, multi-stream contextual sub-core-shape HMMs were suggested where the features estimated from a sliding window form one stream and its horizontal derivative features are the second stream with each stream which different weights. This was applied to the standard Arabic character and provided effective performance.

\section{CONCLUSION}

This article provided a survey of Arabic handwriting recognition. The literature contains considered amount of work with various models based on deep learning and shallow learning. This paper provides an open challenges that need to be tackled in the area of Arabic handwriting recognition. We present them as follows.

1- The need to build a huge dataset that captures different Arabic writing styles is essential to train effective deep learning models.

2- Arabic handwriting in the field of manuscript recognition needs more focus. This is because no available datasets that can help deep learning models for the identification of old Arabic writing.

3- The work of Arabic handwriting recognition has to benefit from languages that have similar characteristics to the Arabic language like the cursive nature such as Farsi, Urdu. 


\section{ACKNOWLEDGMENTS}

The author would like to thank the Mustansiriyah university (www.uomustansiriyah.edu.iq) Baghdad - Iraq for its support in the present work.

\section{REFERENCES}

1. I. Ahmad, and G.A. Fink, "Handwritten Arabic text recognition using multi-stage sub-core-shape HMMs," International Journal on Document Analysis and Recognition (IJDAR). https://doi.org/10.1007/s10032-019-00339-8, 2019.

2. S.A. Firdaus and K. Vaidehi, "Handwritten Mathematical Symbol Recognition Using Machine Learning Techniques: Review", Springer International Publishing. https://doi.org/10.1007/978-3-030-24318-0, 2020.

3. N. Tagougui, M. Kherallah and A.M. Alimi, "Online Arabic handwriting recognition: a survey", https://doi.org/10.1007/s10032-012-0186-8, 2012.

4. T. O. Appear, I. N. Ieee, T. On, and P. Analysis,, Arabic_Character_Recognition_A_Review (阿拉伯语字体辨认).pdf.

5. G. Marcus,. Deep Learning : A Critical Appraisal, pp. 1-27, 2018.

6. Y. Lecun, Y. Bengio, and G. Hinton, Deep learning, Nature, vol. 521 No. 1, pp. 436-444, https://doi.org/10.1038/nature14539, 2015.

7. G. Hinton, Dropout : A Simple Way to Prevent Neural Networks from Overfitting, vol. 15, pp. 1929-1958, 2014.

8. S. Ioffe, and C. Szegedy, Batch Normalization: Accelerating Deep Network Training by Reducing Internal Covariate Shift, 2015.

9. A. Karpathy, and T. Leung, Large-scale Video Classification with Convolutional Neural Networks., 2014.

10. B. Zhou, A. Lapedriza, J. Xiao, A. Torralba, and A. Oliva,. Learning Deep Features for Scene Recognition using Places Database, 2014.

11. B. Alsallakh, and H. Safadi, AraPen : an Arabic Online Handwriting Recognition System, 2006.

12. A. Al-Salman, , Arabic Online Handwriting Recognition : A Survey, 2007.

13. M. Shatnawi, Off-line Handwritten Arabic Character Recognition : A Survey, pp.52-58, 2015.

14. V. Ghods, and M. K. Sohrabi, Online Farsi Handwritten Character Recognition Using Hidden Markov Model, vol. 11, No .2,pp. 169-175. https://doi.org/10.17706/jcp.11.2.169-175, 2015.

15. A. Poznanski, and L., Wolf. CNN-N-Gram for Handwriting Word Recognition, pp. 2305-2314, 2016.

16. S. Mandal, S. R. M. Prasanna, and S. Sundaram, PT. Expert Systems With Applications. https://doi.org/10.1016/j.eswa.2017.12.047, 2017.

17. K. H. Ahmed, "Sequential Segmentation for ArabicHandwriting Using Similarity Measures", International Journal of Civil Engineering andTechnology, vol. 9, No. 13, , pp. 207-213, 2018.

18. B. P. Chacko, V. R. V. Krishnan, G. Raju, and P. B. Anto,. Handwritten character recognition using wavelet energy and extreme learning machine, pp. 149-161. https://doi.org/10.1007/s13042-011-0049-5, 2012.

19. M. Pechwitz, S. S. Maddouri, V. Märgner, (n.d.). IFN / ENIT - DATABASE OF, pp. 1-8, 2002.

20. M. Volker, ICDAR 2011 - Arabic Handwriting Recognition Competition. https://doi.org/10.1109/ICDAR.2011.287, 2011 
21. R. Ahmad, S. Naz, M. Z.Afzal, S. F.Rashid, M., A. Liwicki, \& Dengel, KHATT: a Deep Learning Benchmark on Arabic Script, vol. 1, pp. 1-5, 2017.

22. M. K. Siddhu, "Combining Statistical and Structural Approaches for Arabic Handwriting Recognition", 2019 International Conference on Computer and Information Sciences (ICCIS), 1-6, 2019.

23. A. C. Rouhou, Y. Kessentini, and S. Kanoun, , Hybrid HMM / DNN System for Arabic Handwriting Keyword Spotting (Vol. 4). Springer International Publishing. https://doi.org/10.1007/978-3-030-27202-9, 2019.

24. R. Maalej, N. Tagougui, and M. Kherallah, "Online Arabic Handwriting Recognition with Dropout Applied in Deep Recurrent Neural Networks". Proceedings - 12th IAPR International Workshop on Document Analysis Systems, pp. 417-421. https://doi.org/10.1109/DAS.2016.49, 2016. 